\title{
Perspectival Disagreement
}

\author{
Erich H. Rast \\ New University of Lisbon
}

\begin{abstract}
A phenomenon called perspectival disagreement is laid out and modeled on the basis of modifications to known consensus measures for qualitative representations of preferences and transitive values by binary relations. Cases of perspectival disagreement are of general philosophical interest, because they allow for the possibility that two or more agents judge the value positions of other agents differently even when their assessments are based on the same evidence. Various examples of perspectival disagreement are given, generalizations are discussed, and it is argued that any representation by cardinal utilities also gives rise to some form of perspectivity. Although the examples strongly suggest that this phenomenon occurs in real life, it is concluded in the end that it does not pose any fundamental threat to representations of preferences and values as binary relations. Instead, position-sensitive measures of disagreement ought to be taken as a modeling option for cases in which the relative importance of preference changes matters to an agent.
\end{abstract}

\section{Introducing Perspectivity}

Consider the following scenario. There are four choices for a main dish: salad, goulash, pizza, or a steak. John and Mary have the preferences depicted in Figure 1. Suppose that there are only pizzas and steaks left; then the choice between these two types of dishes may turn out to be more important to Mary than to John, since pizzas and steaks are John's lowest priority, whereas these are on top of Mary's list. Switching from the preference pizza $\succ$ steak to the preference steak $\succ$ pizza, or vice versa, is more important to Mary than to John, because from John's perspective the issue whether they should have a pizza or a steak is not a big deal-he dislikes both of them. This phenomenon was called perspectival disagreement in Rast (2016). In the following sections it is laid out
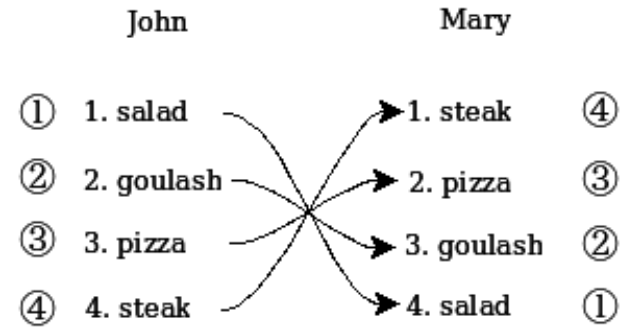

Figure 1: John's and Mary's preferences. 
in more detail and implemented on the basis of modified distance measures that also allow for ties and incomplete preferences. Measures of disagreement are also known as 'consensus measures' in Social Choice Theory. ${ }^{1}$

Why is this important to philosophy? Although values can have a more complicated structure than mere preference relations, the latter can be taken as a basis for modeling value relations which may subsequently be further generalized and relaxed. Perspectival disagreement thus also naturally arises in formal representations of values and the term 'disagreement' can generally be understood as a shortcut for the distance between agents in terms of the nearness or remoteness of the representations of an aspect of a value or a 'value dimension'. This reflects the economic usage of the term such as Debreu (1959) Although there is hardly any agreement in the philosophical community about the adequacy of preference-based approaches to value structure in general, there cannot be much doubt that a proper theory of value structure must be based on similar ordering relations, since many if not most value predicates have a comparative form such as 'better than' which elicits an underlying comparison structure. Although most genuine value predicates express a multidimensional comparative structure, ${ }^{2}$ overall aggregate measures of disagreement are of limited use only because they tend to 'average out' important information. For this reason, even in the most general setting according to which values are neither revealed preferences nor do preferences exhaust an agent's repertoire of values, comparisons between particular value dimensions will be more meaningful than aggregate value comparisons and the former are also based on preferences or at least preference-like relational structures insofar as a value dimension represents an aspect of the comparative form of a value predicate such as 'healthier than', 'better than' or 'braver than'. Perspectivity of preferences might therefore have far-reaching consequences for value representations, as it can help to explain in a purely qualitative setting why two rational agents can disagree about the importance of policies even when they agree on their relative ordering. An example of such a disagreement is given in Section 4.1. If this is plausible for preferences, then it seems reasonable to assume that similar forms of perspectivity may occur in more complex representations of values that are more interesting to philosophers. Another important point is that introducing 3rd-party observers can also lead to the perspectivity of observed disagreement, which is discussed in Section 4.2. Observer-based measures of disagreement can serve to explain in a purely qualitative setting why two rational observers may disagree about the values of two political candidates even though they base their judgments on the very same evidence, for example. That being said, there may be many other forms of value disagreement such as disagreement about weights or about lexicographic orderings of dimensions, and this article only deals with preferences and utility. ${ }^{3}$ Particular philosophical problems of value structure such as the purported nontransitivity of betterness (Schumm 1987; Rachels 1998; Temkin 2012), more general value representations like Hansson (2001), or the problem of

\footnotetext{
${ }^{1}$ See for instance Meskanen \& Nurmi (2006), Garcia-Lapresta et al. (2011) and Erdamar et al. (2014).

${ }^{2}$ See Sassoon (2013) and Sassoon \& Fadlon (2017).

${ }^{3}$ As an anonymous reviewer has pointed out, Kogelmann (fc) and Hun \& Kogelmann (fc) also use the term 'perspectival disagreement' in their recent work. Their use is not directly related to the one laid out in this article and it is worth emphasizing that there can be many other forms of perspectival disagreement, of course. Special about the present approach is that it arises naturally from very minor modifications of existing measures over preference relations.
} 

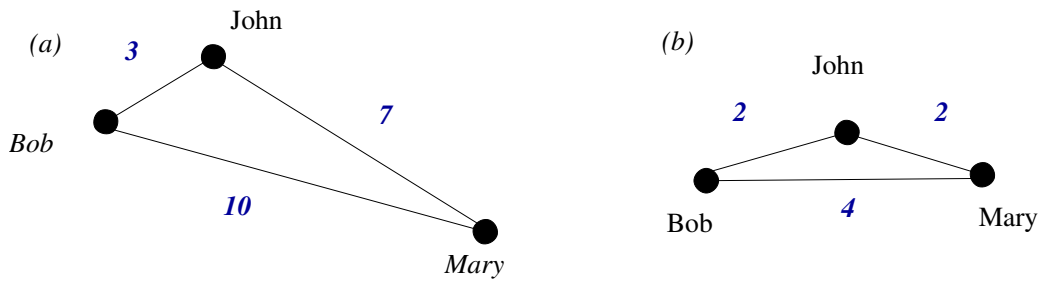

Figure 2: Example of perspectival disagreement among three agents with distances according to (a) positional Footrule and (b) conventional Footrule measure.

parity (Chang 2002) will not be taken into account in what follows for lack of space. $^{4}$

Another example shall illustrate the perspectivity under consideration and motivate the naming choice. Suppose three agents disagree with respect to four items $a, b, c$, and $d$. It does not really matter what items, so let us take them to be the above choices: $a$ pizza, $b$ steak, $c$ goulash, and $d$ salad. This time, John has the preferences $a \succ b \succ c \succ d$, Mary the preferences $b \succ a \succ c \succ d$, and Bob the preferences $a \succ b \succ d \succ c$. Then, in many situations and choice situations in particular, it seems possible that John, as a rational agent, considers his preferences nearer to those of Bob and farther away from Mary, even though their preferences differ only by one switch (adjacent transposition) from his. From the perspective of Mary, on the other hand, John will be nearer to her than to Bob. Finally, from Bob's perspective, Mary's preferences are farther away from his than John's. Figure 2a illustrates this phenomenon, where the numbers next to the arcs are based on a modified version of Spearman's footrule distance measure that will be laid out in Section 3. For comparison, Figure 2b illustrates the ordinary Footrule measure without perspectivity.

There are two natural reactions to such examples. First, one might argue that the connection between the relative importance of items and their position in the preference ordering is only loose. An example that supports this thesis will be discussed in Section 4.1. Another, more far-reaching criticism is that binary relations do not capture cardinal information, but the latter should play a role in measuring disagreement. This is the topic of Section 4.3. For now, notice that the examples become 'intuitively stronger' when there are a lot of items in between the respective preference changes under consideration. Suppose, for instance, that there are 200 other items between item 2 and 3 in the first example; then it seems striking that John really might not care whether he gets his 203rd least favorable or his 204th least favorable dish, whereas Mary might really want to get her very most favorable dish and would be sad to get only her second favorable dish. The positional modifications of distance measures that will be introduced in Section 3 are compatible with this observation, because according to them the perspectival effect is increased whenever more items lie between the respective preference pairs whose potential swap is considered by

\footnotetext{
${ }^{4}$ Hansson (2001) lays out an approach that only requires top-transitivity and weak eligibility and can be considered the weakest possible monist value representation, so it is worth noting that for measures of rational disagreement cycles ought not count. For this application it seems legitimate to collapse cycles into equipreference classes, and as a result the modified consensus measures laid out in this article become applicable. However, further modifications may be needed to adequately deal with edge cases.
} 
an agent. Such examples suggest that perspectival disagreement is possible and occurs in realistic scenarios, and in the following sections it is also laid out how the phenomenon arises from making small, intuitively plausible adjustments to known distance measures. It is not argued, however, that all disagreement about preferences is perspectival. The phenomenon occurs sometimes but not always.

\section{Comparing Preferences}

In this section, known distance measures are laid out which will serve as a basis for the positional variants of Section 3 .

\subsection{Permutations of Strict Preferences}

When preferences are given as strict orders without ties, then every agent's preference will be a permutation of any other agent's preference over the same domain. We write $\pi(p, q)$ for the bijective mapping that expresses the strict ordering of $q$ as a permutation of $p$. For example, for the initial example the mapping $\pi(p, q)=\{$ salad $\mapsto$ steak, goulash $\mapsto$ pizza, pizza $\mapsto$ goulash, steak $\mapsto$ salad $\}$ The corresponding vector that expresses $q$ as a sequence defined by the ordering of $p$ is $\vec{\pi}(p, q)=\langle$ steak, pizza, goulash, salad $\rangle$. We also introduce permutations based on rank vectors of linear preferences. Indexing linear preferences $p$ of size $n$ by the numbers $1,2, \ldots, n$ from most preferred to least preferred element, $q$ may be expressed as a permutation of the natural numbers that represent $p$. This mapping is here written as $M(p, q)$. For example,

$$
\alpha=M(\text { John }, \text { Mary })=\left(\begin{array}{llll}
1 & 2 & 3 & 4 \\
4 & 3 & 2 & 1
\end{array}\right)
$$

is the permutation that represents the initial example. The corresponding vector may be indicated by an arrow symbol; for example, $\vec{\alpha}=\langle 4,3,2,1\rangle$, provided that the underlying preferences are clear from the context. In this particular example, the inverse permutation $M^{-1}$ from Mary's to John's preferences is the same, but this need not be so in general. Suppose John's preferences are again represented by the sequence $\langle 1,2,3,4\rangle$ and Mary's preferences by the vector $\langle 2,3,1,4\rangle$. So the mapping of John's to Mary's preferences is

$$
\beta=M(\text { John }, \text { Mary })=\left(\begin{array}{llll}
1 & 2 & 3 & 4 \\
2 & 3 & 1 & 4
\end{array}\right) .
$$

This time, the inverse mapping is

$$
\beta^{-1}=M(\text { John, Mary })^{-1}=\left(\begin{array}{llll}
1 & 2 & 3 & 4 \\
3 & 1 & 2 & 4
\end{array}\right) .
$$

Note that $M(p, q)=M(q, p)^{-1}$ holds in general, since $M(p, q)$ is defined as a bijective mapping from the rank vector of $p$ to the ranks of the corresponding items in $q$ and both mappings are presumed to be defined over the same set of items. 


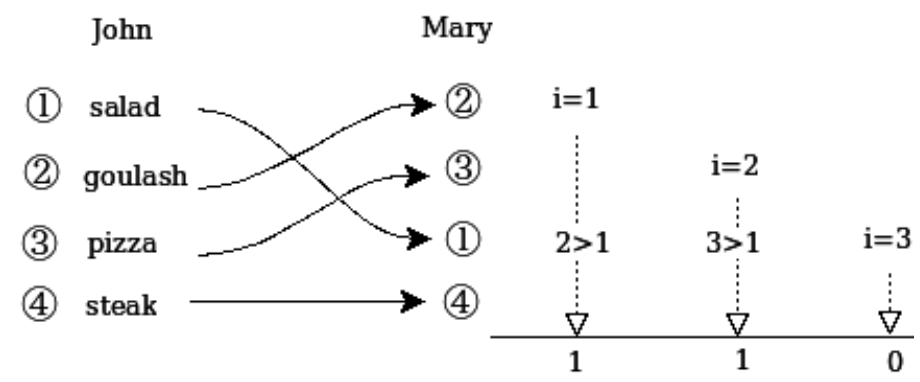

Figure 3: Computing Kendall's Tau.

\subsection{Spearman's Footrule}

Based on Spearman (1904), an easy to calculate measure of disagreement between such preferences is Spearman's footrule; it is computed by summing up the absolute differences between the relative ranks of items, the so-called ' $L_{1}$-distance'. For $\beta$ this is $|1-2|+|2-3|+|3-1|+|4-4|=1+1+2+0=4$. In the present setting, it may be defined as follows:

$$
F(p, q)=\sum_{i=1}^{n}|i-M[p, q](i)|
$$

As the reader may check, the result for the inverse is exactly the same. This property holds in general, i.e., the mapping is symmetric $F(p, q)=F(q, p)$, which follows from the fact that absolute difference is symmetric and from the above observation that $M(p, q)=M(q, p)^{-1}$.

\subsection{Kendall's Tau}

Another popular measure is the inversion number, variants of which are also sometimes called Kendall's Tau or Kemeny measure, following the seminal work of Kendall $(1938 ; 1970)$, Kemeny (1959), and Kemeny \& Snell (1972). ${ }^{5}$ It is based on counting the number of pairs for which two orderings differ. Instead of listing all pairs one may go through the permuted ranks from the most preferred to the one before the least preferred and at each level $i$ add up the number of times one of the following ranks (at positions $j>i$ ) is lower than the rank at $i$. This sum represents the number of flips between adjacent items in the preference ordering (adjacent transpositions) that are needed to transform one preference into the other. Kendall's Tau is often defined as twice the inversion measure, whereas Kendall (1970) defines it as a rank correlation coefficient in the interval $[-1,1]$, but we will ignore these minor differences in what follows. As an example, the inversion measure for the second example is $K(\beta)=1+1=2$, since at $i=1,2>1$ (score 1 ), at $i=2,3>1$ (score 1 ) but at $i=3,1<4$ so this does not count (score 0). Figure 3 illustrates this. For reference, the distances in the initial example of the previous section are the maximum values

\footnotetext{
${ }^{5}$ Diaconis \& Graham (1977) prove that the footrule is always (non-strictly) between the inversion distance and twice the inversion distance. This is important for implementations of these measures, since the footrule measure has better computational complexity than Kendall's Tau.
} 
$K(\alpha)=6$ and $F(\alpha)=4$. To normalize this measure to the interval $[0,1]$, the maximum value for a given size is needed. Closed formulas for this maximum and all other formulae discussed in this article are listed in Appendix B.

Like the footrule measure, Kendall's Tau is symmetric. Moreover, both measures fulfill the other conditions for a proper distance measure, namely coincidence $d(p, q)=0$ iff. $p=q$, and the triangle inequality $d(p, r) \leq d(p, q)+$ $d(q, r){ }^{6}$

\subsection{Preferences With Ties}

There are various ways to generalize distance measures to preorder relations, i.e., weak preference relations that allow for ties. A preorder is a reflexive and transitive binary relation. From a total preorder $a \succeq b$ strict preference can be defined as $a \succ b \Leftrightarrow a \succeq b \quad \& \quad \neg(b \succeq a)$, and an equivalence relation ('equipreference') is defined as $a \sim b \Leftrightarrow a \succeq b \quad \& \quad b \succeq a$.

For Spearman's footrule, tied elements can be assigned a score that results from the average of the ranks of all elements in an apathy class. This 'average tied ranks' approach has already been used by Kendall (1970: Ch. 3) and is studied in detail by Critchlow (1985: 73-77; 130-138), who also discusses its limitations. As an an example of this method, consider the following orderings with ties $a \succ b \succ c \sim d \sim e \succ f \sim g$ and $b \succ c \sim f \succ a \succ e \succ d \sim g$. In this example, the second ordering is given the scores $S(a)=1, S(b)=(3+5) / 2=S(c)=$ $S(d)=S(e)$ and $S(f)=(6+7) / 2=S(g)$. To motivate this way of averaging, notice that the sum of the scores that are averaged equals the ordinary sum $\sum_{i=1}^{n} i=n(n+1) / 2$ whenever the elements of the sequence are not permuted. We write $S^{p}$ for the averaging score vector derived from a preorder $p$ in this way. The generalized footrule then takes into account the averaged rank of each element instead of its simple rank in the permutation. For example, for the above example the footrule is

$$
|1-2.5|+|2-2.5|+|4-6|+|4-1|+|4-5|+|6.5-4|+|6.5-7|=11,
$$

where the values have been obtained from averaged rank vectors on the basis of the following linearized mapping:

$$
\gamma=\left(\begin{array}{lllllll}
1 & 2 & 3 & 4 & 5 & 6 & 7 \\
2 & 3 & 6 & 1 & 5 & 4 & 7
\end{array}\right)
$$

Indices within an apathy class can be ranked in an arbitrary way relative to each other, as long as the ranking method is unique and the same for all preorders under consideration. To ensure this, a natural lexicographic ordering of the items such as alphabetic sorting $a<b<c<\ldots$ or the lesser-than relation for numeric values may be assumed, and such an ordering will be used for obtaining permutations that represent linearizations of preorders from now on. The linearization of a preorder $p$ by some lexicographic ordering $>$ will be written as $p^{>}$in what follows. However, for reasons of space the details of the definition of $S($.$) are omitted. They should be sufficiently clear from the above$ example. The bottom line is that tied items may be linearized in an arbitrary

\footnotetext{
${ }^{6}$ See Kendall (1970) and Diaconis \& Graham (1977) for proofs of the above properties, and Deza \& Deza (2009) for an exhaustive compendium of distance measures, which naturally occur in many different areas of applied mathematics.
} 
way, as long as the linearization method is the same for all preferences under consideration.

As Critchlow (1985: 77-79) lays out, for Kendall's Tau and other measures like the Hamming distance an average tied ranks approach is not readily available. More general methods have been suggested by Critchlow (ibid.), Fagin et al. (2006), but these require relatively complex adjustments or are based on an undesirable ad hoc averaging of the results of applying the measures to different linearizations. In contrast to this, Bogart (1973) has developed and axiomatized a version of Kendall's tau for partial linear orders that can also be used for total and partial preorders. ${ }^{7}$ The measure is computed on the basis of the antisymmetric matrix $A(p, a, b)$. For any pair $(a, b)$ of items in the the union of the domains of two orderings $p, q$ under consideration, this matrix contains 1 if $a \succ b$ according to $p,-1$ if $a \prec b$ according to $p$, and 0 otherwise. ${ }^{8}$ The distance between two orderings $p, q$ is then

$$
B(p, q)=\frac{1}{2} \sum_{a, b \in T}|A(p, a, b)-A(q, a, b)|
$$

for all distinct ordered pairs $a, b$ in the total domain $T$ of both orders. This procedure is illustrated for the above example in Appendix A. For strict orders, Bogart's measure yields exactly the inversion distance, so it is a proper generalization thereof. This measure is particularly well-suited for a notion of overall betterness that not only allows for ties but also for noncomparable items.

\section{Nonsymmetric Position-sensitive Measures}

A first step towards the modeling of perspectival disagreement is to take into account the positions of items, which are thought to reflect their relative importance.

\subsection{The General Case}

When summing up the disagreement value at each level $i$ is multiplied by $(n+1-i)$, thereby assigning a higher score whenever a difference between two orderings occurs at more preferred levels and a lower score when it occurs at a less preferred level. Before adjusting the two sample measures in this way, it is helpful to state a general version of this modification. Suppose the summing function at each level is a linear and increasing function $\Delta(i, p, q)$ that takes the level and both relations as input and yields a value for the difference at level $i$. Writing a measure in the following general form

$$
D(p, q)=\sum_{i=1}^{n} \Delta(i, p, q),
$$

\footnotetext{
${ }^{7}$ Bogart's measure can be derived and motivated independently for preorders by counting ways to slide elements from one adjacent apathy classes to another, where sliding $k$ elements from an apathy class of $r$ elements into an adjacent apathy class of $s$ elements is given the score $k(r+s-k)$. However, we will not go into these details for the sake of brevity.

${ }^{8}$ Taking into account both orderings in the definition is only needed if partial relations are allowed. If an item is ordered by $q$ but not by $p$, then $p$ 's matrix will contain 0 for all comparisons to it, so this treats ties and incompleteness on a par, which might not always be desirable.
} 
a corresponding derived position-sensitive measure will have the form

$$
D_{p o s}(p, q)=\sum_{i=1}^{n} \Delta(i, p, q)(n+1-i) .
$$

The function $\Delta$ needs to be linear, since otherwise cardinal information would be introduced that cannot be derived from the ranking of the items only. This issue will be addressed again in Section 4.3.

\subsection{A Position-sensitive Footrule Measure}

An instance of (3) is the position-sensitive version of Spearman's footrule measure for preorders defined as

$$
F_{p o s}(p, q)=\sum_{i=1}^{n}\left|S^{p}(i)-S^{q}\left(M\left[p^{>}, q^{>}\right](i)\right)\right|(n+1-i),
$$

where the permutation is based on linearizing the respective preorders as indicated above, $S^{p}$ is the scoring function derived from the preorder $p$ by averaging ties in the way indicated above and the index $i$ runs over the linearized preferences.

\subsection{A Position-sensitive Version of Bogart's Measure}

To modify Bogart's measure, it will first be necessary to write the antisymmetric matrix with indices like in the original paper by Bogart (1973: 55-56). Suppose there are two possibly partial preorders $p, q$ and let $p_{i}$ and $q_{i}$ be the $i$-th items in their linearizations respectively. Furthermore, some way of dealing with partiality is required. If $q$ contains items that do not occur in $p$, then we add these to the end of the list of items of $p$ 's linearization in the order in which they occur in $q^{>}$, and likewise for items missing in $q^{>}$that are in $p^{>}$. This ensures that the indices run over the same total domain consisting of the domains of both preorders in case these are partial. When the size of the total domain is $n$, both linearized vectors are of size $n$. Let $A(p)_{i, j}$ be 1 if $p_{i} \succ p_{j},-1$ if $p_{j} \succ p_{i}$ and 0 otherwise. The revised Bogart measure is then defined as

$$
B_{p o s}(p, q)=\sum_{i, j}^{n}\left|A(p)_{i, j}-A(q)_{i, j}\right|(n+1-i) .
$$

\subsection{The Failure of Symmetry}

By giving counter-examples it is easy to show that the new measures are no longer symmetric. For example, for the second case discussed above $(\beta)$, $F_{\text {pos }}($ John, Mary $)=4+3+4+0=11, F_{\text {pos }}($ Mary, John $)=8+3+2+0=13$, $B_{\text {pos }}($ John, Mary $)=16$ and $B_{\text {pos }}($ Mary, John $)=14$.

Since going from $A$ to $B$ should cover the same distance as going from $B$ to $A$, the nonsymmetric variants are no longer proper distance measures. They represent the potential perspectivity of assessments when two or more agents attach differing importance to pairwise comparisons or respective preference changes. 


\section{Generalizations}

In this section, some generalizations are discussed that also address some of the worries mentioned in the beginning. We start by taking a look at a convincing example that requires the introduction of generalized positional weights in Section 4.1. This is followed by a discussion of the philosophically intriguing notion of observer-based disagreement measures in Section 4.2. Finally, the connection between rank-based disagreement and cardinal utilities is discussed in Section 4.3 .

\subsection{Generalized Positional Weights}

Suppose first there are three politicians who disagree about the priorities in response to a major terrorist attack. The options are: 1 - install more CCTV cameras, 2 - increase the budget and training of rapid response police forces, 3 destroy the financial sources of the terrorist group in question, or 4 - raise the budget of intelligence agencies. These are, of course, not exclusive options but for the sake of the argument they may be regarded as being exclusive by taking the ordering as an answer to the question of how to prioritize them. Suppose John's ordering is $\langle 1,2,3,4\rangle$, Mary's is $\langle 1,2,4,3$,$\rangle and Bob's is \langle 2,1,3,4\rangle$. In this case, according to the ordinary footrule measure the distance between John and Mary is the same as the distance between John and Bob, namely 2. The positional footrule, on the other hand, yields 3 as a distance from John to Mary and 7 as the distance from John to Bob. That seems to reflect the nearness of John's to Mary's position from his perspective and the relative distance to Bob from his perspective. ${ }^{9}$ She agrees with him on the topmost items and disagrees with him only with respect to value judgments that he and she consider less important. Again, one might insert arbitrary items between item 2 and 3 to emphasize this divide. This is an ordinary case of perspectival disagreement like the ones already discussed.

However, a similar case with exactly the same preference ordering illustrates that relative importance may sometimes be decoupled from the preference ordering. Suppose that there is compelling evidence that a nation is violating a denuclearization treaty and is in the process of building nuclear weapons. Let the alternatives be: 1 - impose unilateral sanctions, 2 - form a coalition and increase international diplomatic pressure, 3 - threaten the country with military intervention, and 4 - conduct air strikes against weapons manufacturing plants without prior warning. Again, it is not necessary to think of these policies as exclusive options, their ordering may instead be regarded as indicating priorities. Suppose John, Mary and Bob have the same preference orderings as in the previous example. In this case, the claim that the perspectival distance of John to Mary should be lower than that of John to Bob seems less plausible. On the contrary, one might argue that the ordering of items 3 and 4 is more important than the ordering of items 1 and 2, because of the higher stakes associated with former. In the short run, options 1 and 2 are unlikely to lead to loss of lives, whereas 3 and 4 are, and option 4 is also more likely to incur a higher death toll than option 3. For this reason, one might consider a switch of the relative

\footnotetext{
${ }^{9}$ Bear in mind, though, that we are no longer talking about distances in the mathematical sense, neither does the above usage reflect our ordinary understanding of 'distance' at closer scrutiny.
} 
ordering of 3 and 4 more important than a switch of the relative ordering of 1 and 2 when all options are considered at once. To represent such cases, a positional measure must decouple relative importance from the position of an item in a preference ordering, as in the definition

$$
D_{g e n}(p, q)=\sum_{i=1}^{n} \Delta(i, p, q) f(n, i),
$$

where $\Delta(i, p, q)$ is a positive, linear, and increasing function for the score between $p$ and $q$ at level $i$, and $f(x, y)$ is a positive function that attributes some arbitrary importance to item $i$ in dependence of $n$. This function may vary from agent to agent but it also needs to fulfill additional restrictions in order to avoid introducing cardinal comparisons. In the strictest view, $f(.,$.$) is a permutation$ of the sequence $1,2, \ldots, n$, i.e., it assigns to each $i$ a unique integer in $\langle 1, \ldots, n\rangle$. A looser, but intuitively also acceptable condition allows any mapping of indices such that $f(n, x)>0$ and $f(n, x)-f(n, y)=a(x-y)+b$ for positive factor $a$ and constant $b$. Differences between original rankings should be preserved under linear transformations and the result should be positive.

Definition 6 is an instance of a more general class of measures between preorders that have been investigated in statistics and the ranking of search engine data. ${ }^{10}$ However, in this literature great efforts are made to turn the measures into proper distance measures that are symmetric and satisfy other desirable properties of distance functions, but, as the above discussion of examples suggests, symmetry needs not necessarily hold in general when value disagreement is modeled. If relative importance is coupled with the value judgments, then it will vary as dependent on the preference relation that has been chosen as a basis. It is no longer arbitrary whether we take $a$ 's value ordering as a permutation of $b$ 's or vice versa. If, on the other, hand relative importance and value judgments are decoupled like in Definition 6, then they should also be allowed to vary from agent to agent. Symmetry is not guaranteed in either of these cases.

\subsection{Observed Disagreement}

Perspectival disagreement leads to a secondary form of perspectivity when different observers are involved. The idea is to 'filter' one agent's preferences through those of an observer, which has an impact on the judgments about the relative importance of items. Instead of considering the original agent's value system, relative importance is attributed from the perspective of the observer. With a bit of abuse of notation, an observer-relative distance may be schematized as

$$
D_{r}(p, q)=\sum_{i=1}^{n} \Delta(i, \pi(r, p), \pi(r, q))(n+1-i) .
$$

To make this precise, $\Delta$ needs to be redefined to deal with permutations, but since this is a straightforward adjustment the details are left out. The basic idea is to make the summing function at each level depend on the agent's ordering as a permutation of the observer's ordering. The following version of Spearman's

\footnotetext{
${ }^{10}$ See Shieh et al. (2000), Carterette (2009), Chapelle \& Metzler (2009) and Kumar \& Vassilvitskii (2010) amongst others.
} 
footrule for strict orders is an instance of this scheme, where the vector $\vec{\pi}(p, q)$ is used as a synonym for the strict ordering it corresponds to:

$$
F_{r}(p, q)=\sum_{i=1}^{n}|M[r, p](i)-M[p, \vec{\pi}(r, q)](i)|(n+1-i) .
$$

Both $p$ and $q$ are first permuted by the observer's preferences $r$. An observerrelative version of Bogart's measure may be defined in a similar vein. First, the antisymmetric matrix is adjusted for permutations of natural numbers, i.e., $A(p)_{i, j}$ is 1 if $p_{i} \succ_{p} p_{j},-1$ if $p_{j} \succ_{p} p_{i}$, and 0 otherwise. To deal with incompleteness it is assumed that the indices $i, j$ may become larger than the size of the permutation for those items that are in another preference but not the one represented by $p$. Likewise, the revised matrix is 0 whenever items $i$ and $j$ are tied in the original ordering that $p$ partially represents. The measure is then defined as

$$
B_{r}(p, q)=\sum_{i, j}^{n}\left|A(\pi[r, p])_{i, j}-A(\pi[r, q])_{i, j}\right|(n+1-i),
$$

where $n$ is the size of the union of the domains over which the original ordering relations are defined.

The above method does, however, presume that the orders are strict, and it may be argued that attempting to adjust these definitions to preorders directly is of limited use, for an average tied ranks approach is not necessarily meaningful in this setting and at least a naive method of dealing with all possible linearizations leads to a combinatorial explosion. Take the preferences with ties $p=\langle 2,3,\{1,4\}\rangle, q=\langle 3,\{1,4\}, 2\rangle$, and an observer $r=\langle\{1,3\}, 2,4\rangle$. Suppose $\circ$ stand for a generalized function combination operation that yields all possible combinations of two preorders whose ties are linearized in all possible ways respectively. Then $p \circ r$ results in four permutations $\langle 2,1,3,4\rangle$, $\langle 2,4,3,1\rangle,\langle 1,2,3,4\rangle$, and $\langle 4,2,3,1\rangle$. Another four permutations correspond to $q \circ r:\langle 3,1,4,2\rangle,\langle 3,4,1,2\rangle,\langle 1,3,4,2\rangle$, and $\langle 4,3,1,2\rangle$. So already for this simple example an averaging approach would require sixteen calculations. In the worst case, when all three preorders of size $n$ only contain ties, $(n !)^{4}$ calculations are needed, making this method impractical for large preorder relations with many

\begin{tabular}{|c|c|}
\hline Agent & Permutation \\
\hline Mary & 356412 \\
\hline John & 521436 \\
\hline Bob & 154362 \\
\hline Homer & 362154 \\
\hline Lisa & 451326 \\
\hline
\end{tabular}
ties. $^{11}$

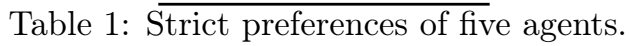

Interesting about the above variants is that they become symmetric again for the same observer and, perhaps more interestingly, if observers disagree among each other, then they may come to radically different assessments of the perceived differences between the preferences of other persons.

\footnotetext{
${ }^{11}$ There are $n$ ! ways to arrange $n$ tied elements into a sequence of elements, and each of these may be combined with $n$ ! ways of arranging the observer's tied elements. By the product rule, there are therefore $n ! \cdot n$ ! possibilities forl fombining the observer's preorder with that of an agent, hence by another application of the product rule $(n ! \cdot n !) \cdot(n ! \cdot n !)$ total calculations are necessary for the worst case. Although it is likely possible to compute this particular worst case directly, a shortcut does not seem to be available in general.
} 
As an example, consider the strict preferences represented by the permutations in Table 1. In this example, for Homer the observed footrule distance between Mary and John is 72 and between Mary and Bob the distance is 29, so from his point of view Mary disagrees much more with John than she disagrees with Bob. In contrast to this, Lisa observes the distances 43 and 45 respectively, so from her point of view, Mary almost agrees as much with Bob as she agrees with John. Even though Homer and Lisa both have full knowledge of the preferences of Mary, John, and Bob, and may be taken to base their initial assessments of these preferences on the same evidence, they evaluate them differently.

\subsection{Disagreement and Cardinal Utility}

The methods of Section 2 were designed to deal with ordinal rankings, and one might wonder about the appropriateness of these methods for representations of preferences that are endowed with additional cardinal information. To address this question, we look at utility functions defined in the usual way. Let $u($. a function from items to real numbers such that the following representation condition is fulfilled:

$$
\begin{aligned}
& u(x)>u(y) \Leftrightarrow x \succ y \\
& u(x)=u(y) \Leftrightarrow x \sim y
\end{aligned}
$$

Based on this representation the difference between ordinal and cardinal utilities can be characterized by invariance conditions. Consider two utility functions $u($.$) and v($.$) that represent a preorder \succeq$ in the sense of (10). Then $u($.$) is$ ordinal whenever $u(x)=F(v(x))$ holds for any strictly increasing function $F$. In other words, if $u($.$) is ordinal, then any strictly increasing transformation$ of it will represent the same underlying preferences. It is well-known that differences like $u(x)-u(y)$ cannot be compared with each other and are, in this sense, not generally meaningful in this setting, since $F$ may not be linear. Only the ordering counts and the utility function is merely an alternative way of representing the underlying complete preorder. ${ }^{12}$ In contrast to this, if additional cardinal information is added, then the equation $u(x)=a v(x)+b$ must be fulfilled for positive $a$ and some $b .^{13}$ In this case, the utility function does not only represent an underlying preorder, but also differences between levels, making comparisons like $u\left(x_{1}\right)-u\left(x_{2}\right)>u\left(y_{1}\right)-u\left(y_{2}\right)$ meaningful. ${ }^{14}$

Let us suppose that we are dealing with these richer cardinal representations. Then it seems at first glance that the above measures are inadequate, because we can now directly take into account differences between adjacent items and even between items farther apart. For instance, if in the meal example $u(c)-u(d)>$ $u(a)-u(b)$ holds for John, then it would be seemingly absurd to claim that John is nearer to Bob than to Mary from his perspective, because from his perspective $c$ and $d$ are farther apart than $a$ and $b$.

This view is principally correct if the preference differences and levels are based on objective, extensively measurable quantities like salary, price, temperature,

\footnotetext{
${ }^{12}$ If the underlying preorder is not complete, the representation conditions in (10) need to be weakened to implications from right to left. See Roubens \& Vincke (1985: 48/9).

${ }^{13}$ See Hammond (1998: 151). This observation goes back to von Neumann \& Morgenstern (1944) in response to Pareto (1906).

${ }^{14} \mathrm{We}$ assume countable and even finite domains for simplicity, as it is well-known that additional assumptions are needed for uncountable domains.
} 
or size, because in this case the respective scale allows for a direct comparison of distances between pairs. However, this does not imply that cardinal information can be taken as a basis of measures of value disagreement in general. It may very well be possible to ascribe utility levels and corresponding difference comparisons to an agent in a value dimension that is not directly measurable, without thereby subscribing to the more controversial view that interpersonal comparisons of utility differences are meaningful in this dimension. For example, an agent might consider one alternative for action 'much better' than another alternative in some dimension of betterness under a certain covering value, but this does not imply that his subjective feeling about 'how much better' the one item is in comparison to the other can be compared to another agent's feeling in that respect. ${ }^{15}$ Even if utilities are cardinal for individual agents, an additional step is required to justify their interpersonal comparability. This issue has been hotly debated for decades in the philosophy of economics and cannot be addressed at any sufficient level of detail here. But even without entering this debate, a few observations can be made: Firstly, utility differences can be taken as a basis of disagreement measures only if there is a sound justification as to why they can be compared across individuals. If so, they will give rise to a form of perspectival disagreement similar to the one that has been discussed in Section 4.1. In other words, cardinal utilities that are fully comparable across individuals may lead to perspectival disagreement, too. Secondly, insofar as cardinal utilities may also represent preferences or values-qua-preferences in the sense of (10), the above measures may serve as an indicator even if the utilities are cardinal, but then it must be made plausible why a potential conflict between the cardinal information and the rank-based notions of relative importance of the positional variants is harmless. Perhaps this is possible sometimes when the measure is used in a comparison context in which cardinal information plays no role, even though it is present. Many social choice situations are of this type, for example. ${ }^{16}$ Thirdly, if the utilities represent preferences in the sense laid out above, then the measures may, of course, also just be taken as indicators in addition to methods that rely on cardinal information.

\section{Conclusion}

The examples laid out above suggest that positional distance measures can be a useful tool for modeling perspectival disagreement. However, it seems that their importance ought not be overstated. Positional measures have been derived as a natural generalization from ordinary symmetric distance measures by adding positional weights, and the perspectivity of preference disagreement has been made plausible on the basis of relatively realistic examples, yet from the above discussion it should be apparent that there is no reason to believe that perspectivity constitutes a principal problem for the comparison of preference

\footnotetext{
${ }^{15}$ Some of the terminology is borrowed from Chang (2015), who uses the term 'covering value' to describe an overarching value under which comparisons are made, such as well-being or happiness.

${ }^{16}$ Arguably, this is only true for the classical approach of Arrow (1951), which was devised as an ordinal method from the start. Although most common voting methods are rank-based and ordinal, it is in principle possible to make use of cardinal information for voting, in case of which one has to switch to a 'social welfare functional' approach pioneered by Sen (1970), d'Aspremont \& Gevers (1977) and others.
} 
representations. This stronger position would require an additional argument to establish the claim that the observation of other people's preferences (viz. values, formalized in a preference-based setting) is principally filtered through the relative importance constituted by an observer's preferences, and this argument seems hard to make, since proper distance measures are readily available for making objective comparisons. Hence, perspectival disagreement ought to be merely taken as a tool for representing cases when agents attach more importance to items higher than to items lower in the preference ordering and when cardinal information is not available or does not play a justifiable role for the comparison. If these conditions are fulfilled, then perspectivity is unavoidable, and attempts to eliminate it and make the positional measure symmetric again would miss the point. The modified positional Bogart's measure provides a very general method for representing such cases when preorder relations or incomplete strict orders are taken as a basis of the underlying preference model.

\section{Acknowledgements}

I am grateful to the Portuguese Foundation for Science and Technology (FCT) for the individual grant $\mathrm{SRFH} / \mathrm{BPD} / 84612 / 2012$ and the project grant PTDC/MHCFIL/0521/2014 under which work on this article was conducted. Many thanks also go to members of the ArgLab Colloqium at the IFILNOVA Institute of Philosophy of the New University of Lisbon, to members of the FCT project "Values in Argumentative Discourse", and to Tad White for helpful commentaries on earlier drafts of this article. 


\section{Appendix A: An Example of Calculating Bogart's Measure}

Calculation of Bogart's distance measure for John's preferences $a \succ b \succ c \sim d \sim$ $e \succ f \sim g$ and Mary's preferences $b \succ c \sim f \succ a \succ e \succ d \sim g$. John's matrix is listed in Table 2 and Mary's in Table 3.

\begin{tabular}{rrrrrrrr}
\hline & $\mathrm{a}$ & $\mathrm{b}$ & $\mathrm{c}$ & $\mathrm{d}$ & $\mathrm{e}$ & $\mathrm{f}$ & $\mathrm{g}$ \\
\hline $\mathrm{a}$ & 0 & 1 & 1 & 1 & 1 & 1 & 1 \\
$\mathrm{~b}$ & -1 & 0 & 1 & 1 & 1 & 1 & 1 \\
$\mathrm{c}$ & -1 & -1 & 0 & 0 & 0 & 1 & 1 \\
$\mathrm{~d}$ & -1 & -1 & 0 & 0 & 0 & 1 & 1 \\
$\mathrm{e}$ & -1 & -1 & 0 & 0 & 0 & 1 & 1 \\
$\mathrm{f}$ & -1 & -1 & -1 & -1 & -1 & 0 & 0 \\
$\mathrm{~g}$ & -1 & -1 & -1 & -1 & -1 & 0 & 0 \\
\hline
\end{tabular}

Table 2: John's antisymmetric matrix.

\begin{tabular}{rrrrrrrr}
\hline & $\mathrm{a}$ & $\mathrm{b}$ & $\mathrm{c}$ & $\mathrm{d}$ & $\mathrm{e}$ & $\mathrm{f}$ & $\mathrm{g}$ \\
\hline $\mathrm{a}$ & 0 & -1 & -1 & 1 & 1 & -1 & 1 \\
$\mathrm{~b}$ & 1 & 0 & 1 & 1 & 1 & 1 & 1 \\
$\mathrm{c}$ & 1 & -1 & 0 & 1 & 1 & 0 & 1 \\
$\mathrm{~d}$ & -1 & -1 & -1 & 0 & -1 & -1 & 0 \\
$\mathrm{e}$ & -1 & -1 & -1 & 1 & 0 & -1 & 1 \\
$\mathrm{f}$ & 1 & -1 & 0 & 1 & 1 & 0 & 1 \\
$\mathrm{~g}$ & -1 & -1 & -1 & 0 & -1 & -1 & 0 \\
\hline
\end{tabular}

Table 3: Mary's antisymmetric matrix.

Summing up all distinct pairs $\mid \mathrm{A}($ John, $\mathrm{x}, \mathrm{y})-\mathrm{A}($ Mary, $\mathrm{x}, \mathrm{y}) \mid$ yields the result in Table 4.

\begin{tabular}{crrr}
\hline Pairs & John & Mary & Result \\
\hline a,b & 1 & -1 & 2 \\
a,c & 1 & -1 & 2 \\
a,d & 1 & 1 & 0 \\
a,e & 1 & 1 & 0 \\
a,f & 1 & -1 & 2 \\
a,g & 1 & 1 & 0 \\
b,c & 1 & 1 & 0 \\
b,d & 1 & 1 & 0 \\
b,e & 1 & 1 & 0 \\
b,f & 1 & 1 & 0
\end{tabular}




\begin{tabular}{crrr}
\hline Pairs & John & Mary & Result \\
\hline $\mathrm{b}, \mathrm{g}$ & 1 & 1 & 0 \\
$\mathrm{c}, \mathrm{d}$ & 0 & 1 & 1 \\
$\mathrm{c}, \mathrm{e}$ & 0 & 1 & 1 \\
$\mathrm{c}, \mathrm{f}$ & 1 & 0 & 1 \\
$\mathrm{c}, \mathrm{g}$ & 1 & 1 & 0 \\
$\mathrm{~d}, \mathrm{e}$ & 0 & -1 & 1 \\
$\mathrm{~d}, \mathrm{f}$ & 1 & -1 & 2 \\
$\mathrm{~d}, \mathrm{~g}$ & 1 & 0 & 1 \\
$\mathrm{e}, \mathrm{f}$ & 1 & -1 & 2 \\
$\mathrm{e}, \mathrm{g}$ & 1 & 1 & 0 \\
$\mathrm{f}, \mathrm{g}$ & 0 & 1 & 1 \\
Total: & & & 16 \\
\hline 4: Computing
\end{tabular}

Table 4: Computing the Bogart distance.

\section{Appendix B: Maximum Values}

Let $n$ be the the cardinality of the union of the domains of the respective preorders for Bogart's measure and its positional variant, the size of the linearized preorders for the other measures. The maximum values in terms of $n$ are:

- Footrule Distance (Diaconis \& Graham 1977: 264): $\left\lfloor n^{2} / 2\right\rfloor$.

- Kendall's Tau (Kendall 1970), Bogart Distance (Bogart 1973: 64): $n(n-1)$.

- Positional Footrule (Tad White, p.c.): $\frac{1}{2} a(-1+a-n)(a-n)+\frac{1}{6}(-a-$ $\left.3 a^{2}+4 a^{3}+3 a n-9 a^{2} n+6 a n^{2}\right)$, where $a=\operatorname{round}(3 n / 7)$.

- Positional Bogart (OEIS A002378 17$): 2 k n(k+2) / 3$, where $k=\max (n-$ $1,0)$.

\section{References}

Arrow, K. J. (1951). Social Choice and Individual Values. Yale University Press, New Haven.

d'Aspremont, C. \& Gevers, L. (1977). Equity and the Informational Basis of Collective Choice. Review of Economic Studies, Vol. 44, 199-209.

Bogart, K. P. (1973). Preference structures I: Distances between transitive preference relations. Journal of Mathematical Sociology, 49-67.

Carterette, B. (2009). On Rank Correlation and the Distance Between Rankings. In Allan, J. \& Aslam, J. (Eds.): Proceedings of the 32nd international ACM SIGIR conference on Research and development in information retrieval, ACM, 436-443.

${ }^{17}$ See Sloane (2017), https://oeis.org/A002378. 
Chang, R. (2015). Value Incomparability and Incommensurability. In Chang, R. Hirose, I. \& Olson, J. (Eds.): The Oxford Handbook of Value Theory, Oxford University Press, 205-224.

Chang, R. (2002). The Possibility of Parity. Ethics, Vol. 112, 669-688.

Chapelle, O. \& Metzler, D. (2009). Expected Reciprocal Rank for Graded Relevance. In Cheung, D. \& Song, I.-Y. (Eds.): Proceedings of the 18th ACM conference on Information and knowledge management, ACM, 621-630.

Critchlow, D. E. (1985). Metric Methods for Analyzing Partially Ranked Data. Springer.

Debreu, G. (1959). Theory of Value: An Axiomatic Analysis of Economic Equilibrium. Yale University Press.

Deza, M. M. \& Deza, E. (2009). Encyclopedia of Distances. Springer.

Diaconis, P. \& Graham, R. L. (1977). Spearman's Footrule as a Measure of Disarray. Journal of the Royal Statistical Society. Series B (Methodological), Vol. 39, 262-268.

Erdamar, B.; García-Lapresta, J. \& Pérez-Román, D. (2014). Measuring consensus in a preference-approval context. Information Fusion, Vol. 17, 14-21.

Fadlon, J. \& Sassoon, G. Weidman (2017). The role of dimensions in classification under predicates predicts their status in degree constructions. Glossa, Vol. 2 (1), No. 42, 1-40.

Fagin, R.; Kumar, R.; Mahdian, M.; Sivakumar, D. \& Vee, E. (2006). Comparing Partial Rankings. Journal of Discrete Mathematics, Vol. 20, 628-648.

Garcia-Lapresta, J. L.; Pérez-Román, D. \& Herrera-Viedma, E. (2011). Measuring Consensus in Weak Orders. In Herrera-Viedma, E.; García-Lapresta, J.; Kacprzyk, J.; Fedrizzi M., N. H. \& Zadrozny, S. (Eds.): Consensual Processes, Springer, 213-234.

Hamming, R. W. (1950). Error detecting and error correcting codes. Bell System Technical Journal, No. 29, 147-160.

Hammond, P. J. (1998). Objective Expected Utility. In Barbera, S.; Hammond Peter, J. \& Seidl, C. (Eds.). Handbook of Utility Theory, Vol. 1, Kluwer, 143-211.

Hansson, S. O. (2001). The Structure of Values and Norms. Oxford UP.

Hun, C. \& Kogelmann, B. (fc.). Diversity and rights: a social choice-theoretic analysis of the possibility of public reason. Synthese, forthcoming, Online First DOI 10.1007/s11229-018-1737-4.

Kemeny, J. (1959). Mathematics without Numbers. Daedalus 88, 577-591. 
Kemeny, J. \& Snell, J. L. (1972). Mathematical Models in the Social Sciences. MIT Press.

Kendall, M. G. (1938). A New Measure of Rank Correlation. Biometrika, Vol. 30, 81-93.

Kendall, M. G. (1970). Rank Correlation Methods. Griffin. (First published 1948.)

Kogelmann, B. (fc.). What we choose, what we prefer. Synthese, forthcoming, Online First DOI 10.1007/s11229-017-1369-0.

Kumar, R. \& Vassilvitskii, S. (2010). Generalized Distances between Rankings. In Proceedings of the $W W W 2010$ Conference, 571-579.

Meskanen, T. \& Nurmi, H. (2006). Distances from Consensus: A Theme and Variations Mathematics and Democracy. In Simeone, B. \& Pukelsheim, F. (Eds.): Recent Advances in Voting Systems and Collective Choice, Springer, 117-132.

von Neumann, J. \& Morgenstern, O. (1944). Theory of Games and Economic Behavior. Princeton UP.

Rast, E. H. (2016). Modeling Value Disagreement. Erkenntnis, Vol. 81, No. 4. pp. 853-880.

Pareto, V. (1906). Manuale di Economia Politica. Societa Editrice Libraria.

Rachels, S. (1998). Counterexamples to the transitivity of better than. Australasian Journal of Philosophy, Vol. 76, No. 1, pp. 71-83.

Roubens, M. \& Vincke, P. (1985). Preference Modelling. Springer.

Sassoon, G. Weidman (2013). A Typology of Multidimensional Adjectives. Journal of Semantics, Vol. 30, 335-380.

Sen, A. K. (1970). Collective Choice and Social Welfare. Holden-Day.

Schumm, G. F. (1987). Transitivity, Preference and Indifference. Philosophical Studies, Vol. 52, 435-437.

Shieh, G. S. ; Zhidong, B. \& Tsai, W.-Y. (2000). Rank Tests for Independence With a Weighted Contamination Alternative. Statistica Sinica 10, 577-593.

Sloane, N. J. A. (Ed.). (2017). The On-Line Encyclopedia of Integer Sequences, published electronically at https://oeis.org.

Spearman, C. (1904). The Proof and Measurement of Association Between Two Things. The American Journal of Psychology, Vol. 15, 72-101. 
Temkin, L. S. (2012). Rethinking the Good: Moral Ideals and the Nature of Practical Reasoning. Oxford University Press . 